quarterly exams, taking into account exams realised during each period for syphilis, gonorrhoea and Chlamydia, and up to and including each period for HIV.

Results Overall, the number of FSW accessing VICITS annually increased from 555 to 2557 (361\%) during the period. At the longest running sites (1 and 2), a plateau or decline of up to $13 \%$ annually was observed after year 3 (2010 and 2011, respectively). In parallel, retention rates declined from $51 \%$ to $41 \%$ at site 1 (2007-2011), from $72 \%$ to $41 \%$ at site 2 (2008-2011), and increased from $14 \%$ to $35 \%$ at site 3 (2010-2011). HIV prevalence varied from $0.6 \%$ to $4.8 \%$ and prevalence of Chlamydia, the most common STI, from $1.9 \%$ to $8.5 \%$, with differences across sites but not over time.

Conclusions VICITS achieved rapid scale-up and has identified local differences in relatively stable infection prevalence that can help to prioritise prevention programming among FSW. However, a better understanding of the causes of low follow-up by new users is needed to improve interpretation of surveillance findings.

\section{P3.399 WHO ARE REGULAR SEX PARTNERS OF FEMALE SEX WORKERS IN BOBO-DIOULASSO, BURKINA FASO?}

doi:10.1136/sextrans-2013-051184.0852

II Konate, ' $\mathrm{A}$ Ouédraogo, 'W W Bazié, 'A Sanon, 'H A Sawadogo, ${ }^{2} \mathrm{~T}$ T Traoré, 'N Méda, ${ }^{3} \mathrm{P}$ Mayaud, ${ }^{4} \mathrm{P}$ Van De Perre, ${ }^{4} \mathrm{~N}$ Nagot. ' $C e n t r e ~ M U R A Z$, Bobo-Dioulasso, Burkina Faso; ${ }^{2}$ Centre de Recherche International en Santé, University of Ouagadougou, Burkina Faso, ${ }^{3}$ LSHTM, London, UK; ${ }^{4}$ INSERM U 1058, Montpellier, France

Background In Burkina Faso, female sex workers (fsw) remain a core group for HIV transmission. Unfortunately fsw use condoms less consistently with their regular sex partners (RSP). Yet these RSP are not taken into account in interventions. Knowledge of their characteristics will help develop strategies to involve them in the fight against HIV. This study aimed to describe the sociodemographic and behavioural characteristics of RSP of fsw in BoboDioulasso.

Methods The study included baseline data of the Yèrêlon cohort (fsw cohort) formed between December 2003 and March 2011. A standardised questionnaire was administered face to face to fsw to gather information on their RSP. Data were entered into Access and analysed with stata11.

Results We included 918 women (seaters, roamers, bar waitresses, sellers, cabarets and others). The median number of RSP pers fsw was 2 (range 0-5). These RSP were considered by the fsw as "boyfriends" (52.9\%) and the relationship lasted less than a year (39.2\%). They were of unknown age $(62.5 \%)$, single $(52.7 \%)$, national of Burkina Faso (94.9\%), residing in Bobo (89.3\%), travelling often $(33.0 \%)$, using condoms consistently $(28.4 \%)$. RSP of fsw had secondary or higher level of education (55.2\%), and another regular sex partners $(68.2 \%)$

Conclusion In relation to these characteristics, we are all concerned. The RSP approach will be difficult. Due to their position as "bridge group" between fsw and the general population, a qualitative investigation is necessary with fsw to develop a targeted strategic approach.

\section{P3.400 COMPARING THE UTILITY OF CONDOM AND HIV PRE- EXPOSURE PROPHYLAXIS (PREP) USE AMONG FSWS}

doi:10.1136/sextrans-2013-051184.0853

Z Mukandavire, P Vickerman. Social and Mathematical Epidemiology Group, London School of Hygiene and Tropical Medicine, London, UK

Background In many settings, HIV transmission is thought to be driven by high-risk groups, thus interventions targeting these groups could have a significant effect on the dynamics of the disease. As efforts to integrate PreP into the package of HIV prevention strategies continues, its impact in reducing HIV prevalence among female sex workers (FSWs) has rarely been assessed, and no studies have factored in the importance of 'pimps' (other men involved in sex industry that have sex with FSWs but use condoms infrequently). It is crucial to understand how the protection provided by PreP compares with condom use - a prevention strategy that has been shown to be effective in reducing HIV transmission amongst FSWs.

Methods A simple dynamic HIV transmission model which consists of FSWs, their clients and pimps was developed and parameterised using data from heterosexual settings. Analytical and numerical tools were used to explore the protection provided by condom use amongst FSWs, clients and/or pimps and PreP use among FSWs.

Results By deriving coverage levels at which PreP provides the same reduction in endemic HIV prevalence as different consistencies of condom use, we noted that the relative effectiveness of PreP is generally less than condoms $(10 \%$ increase in condom consistency is equivalent to $\sim 20 \%$ increase in PreP coverage for a FSW population with $40 \%$ HIV prevalence), but increases with baseline FSW HIV prevalence and once the effect of pimps are incorporated.

Conclusion Our results suggest that in most scenarios it may be better to scale up activities to increase condom use amongst FSWs than introduce PreP. However, the benefits of PreP become more favourable in settings with high FSW HIV prevalence, where condom use is already high and where other men are involved in sex work, but use condoms infrequently and contribute considerably to HIV transmission.

\section{P3.401 IMPLEMENTING FOR RESULTS: PROGRAM ANALYSIS OF THE HIV/STI INTERVENTIONS FOR SEX WORKERS IN BENIN}

doi:10.1136/sextrans-2013-051184.0854

'G Batona, ${ }^{21}$ Sémini, ${ }^{1} \mathrm{M}$ Gagnon, ${ }^{3} \mathrm{~F}$ Guedou, ${ }^{1,4} \mathrm{M}$ Alary. ${ }^{1}$ Université Laval, Québec, OC, Canada; 'World Bank, Washington, WA, United States; ${ }^{3}$ Dispensaire IST, Cotonou, Benin; ${ }^{4} \mathrm{CHU}$ de Québec, Québec, OC, Canada

Background Benin is among the pioneering countries that prioritised HIV prevention for female sex workers (FSWs) early on. Interventions were scaled-up, but since 2008, indicators of coverage are far from satisfactory.

Objective To better understand implementation and how to render service delivery for FSWs efficient and effective.

Methods Quantitative and qualitative methods were utilised to collect data for 2010-2011 in nine regions of Benin. A conceptual framework based on an evaluative approach was used to analyse the technical efficiency of the implementation of ongoing interventions. The Avahan (in India) and SIDA-3 (in West Africa) projects served as benchmark comparison for the programme design and implementation modalities. A top-down approach cost analysis reviewed costs in four categories: NGO activities, clinical, monitoring, and management. The output is the number of FSWs seeking STI care at user-friendly STI Clinics (SCs).

Results Allocation of funds was not proportionate to FSW needs across regions. Only 5 of $41 \mathrm{SCs}$ were fully functional. Free condom distribution covers only $10 \%$ of needs. Funding gaps resulted in extended interruptions of services. The NGO cost per FSW seeking STI care varied from US $\$ 7$ to more than US $\$ 2435$ from one SC to the other, with an overall of US\$61 per FSW visit. In high-volume SCs, the overall NGO cost per FSW visit was US\$28.5 (range: US\$7US $\$ 103$. This was significantly lower than in low-volume clinics $(p=0.039)$, where the overall NGO cost per FSW visit was US\$481.7 (range: US\$113-US\$2435. The average total cost (NGO, clinical, surveillance and management) per FSW visit to two high-volume SCs were between US\$28.6 and US\$46.9, similar to the unit costs of the Avahan project.

Conclusion The study revealed deficiencies in programme design and implementation. A national framework defining an appropriate mix of interventions, management structure and operational standards is required to guide rigorous implementation. 